\title{
Erratum: Slicing the Fock space for state production and protection [Phys. Rev. A 90, 033840 (2014)]
}

\author{
R. F. Rossetti, G. D. de Moraes Neto, F. O. Prado, F. Brito, and M. H. Y. Moussa \\ (Received 8 June 2016; published 20 June 2016)
}

DOI: 10.1103/PhysRevA.93.069904

In our paper we presented a protocol to engineer interactions confined to subspaces of the Fock space in trapped ions: upperand lower-bounded Jaynes-Cummings (JC) and anti-Jaynes-Cummings (AJC) interactions, acting upon Fock subspaces ranging from $|0\rangle$ to $|M\rangle$ and $|N\rangle$ to $\infty$, respectively, and sliced JC and AJC interactions, confined to Fock subspaces ranging from $|M\rangle$ and $|N\rangle$, whatever $M<N$. We have considered a single laser beam to couple the electronic and vibrational degrees of freedom of the trapped ion in the resolved sideband regime where the detuning between the electronic transition frequency $\omega_{0}$ and the laser field $\omega$ is an integer $k$ multiple of the vibrational frequency $v$, i.e., $k=\left(\omega_{0}-\omega\right) / \nu$. By tuning the laser beam to the first red or blue sideband and working to second order in the Lamb-Dicke parameter $\eta$, we derived the interactions,

$$
H_{k= \pm 1}=\chi(\eta)\left[\mathbf{A}(\eta) \sigma_{ \pm}+\mathbf{A}^{\dagger}(\eta) \sigma_{\mp}\right],
$$

where $\chi(\eta)=\eta\left(1-\eta^{2} / 2\right) \Omega$ and $\mathbf{A}(\eta)=\left[1-\eta^{2} a^{\dagger} a / 2\right] a, \Omega$ being the Rabi frequency and $\phi$ being the phase of the laser field. We have thus neglected all terms proportional to $\left(a^{\dagger}\right)^{m} a^{m}$ with $m \geqslant 2$ in the field operator $\mathbf{A}(\eta)$, which is completely correct for the case of $N=1$ where all the corrections $(m \geqslant 2)$ turn to be null. However, for all other values of $N$ the operator $\mathbf{A}(\eta)$ becomes a good approximation only for time intervals around $\left(\chi \bar{n}^{2}\right)^{-1}, \bar{n}$ being the mean excitation of the vibrational mode, thus compromising our state protection scheme. In order to overcome this problem we have to consider two laser fields, both tuned to the first red or blue sideband and completely out of phase of each other, leading to the interaction,

$$
H_{k= \pm 1}=\Omega_{1}\left[\mathbf{A}\left(\eta_{1}, \eta_{2}\right) \sigma_{ \pm}+\mathbf{A}^{\dagger}\left(\eta_{1}, \eta_{2}\right) \sigma_{\mp}\right],
$$

where $\Omega_{j}=\eta_{j}\left(1-\eta_{j}^{2} / 2\right) \bar{\Omega}_{j}$ with $j=1,2$, and

$$
\mathbf{A}\left(\eta_{1}, \eta_{2}\right)=a^{\dagger}\left[1-\frac{\Omega_{2}}{\Omega_{1}}-\frac{1}{2 !}\left(\eta_{1}^{2}-\frac{\Omega_{2}}{\Omega_{1}} \eta_{2}^{2}\right) a^{\dagger} a+\frac{1}{2 ! 3 !}\left(\eta_{1}^{4}-\frac{\Omega_{2}}{\Omega_{1}} \eta_{2}^{4}\right)\left(a^{\dagger}\right)^{2} a^{2}+\cdots\right],
$$

$\bar{\Omega}_{j}$ being the Rabi frequency and $\eta_{j}$ being the Lamb-Dicke parameter. By adjusting the laser parameters such that

$$
\frac{1}{2 !}\left(\eta_{1}^{2}-\frac{\Omega_{2}}{\Omega_{1}} \eta_{2}^{2}\right) a^{\dagger} a=1-\frac{\Omega_{2}}{\Omega_{1}} \approx 0.01,
$$

and cooling the trapped ion into the Lamb-Dicke regime with $\eta_{1}, \eta_{2} \ll 1$, we conclude that the remaining terms in the expansion of the operator $\mathbf{A}\left(\eta_{1}, \eta_{2}\right)$, proportional to $\left(a^{\dagger}\right)^{m} a^{m}$ with $m \geqslant 2$, may now be neglected to a good approximation
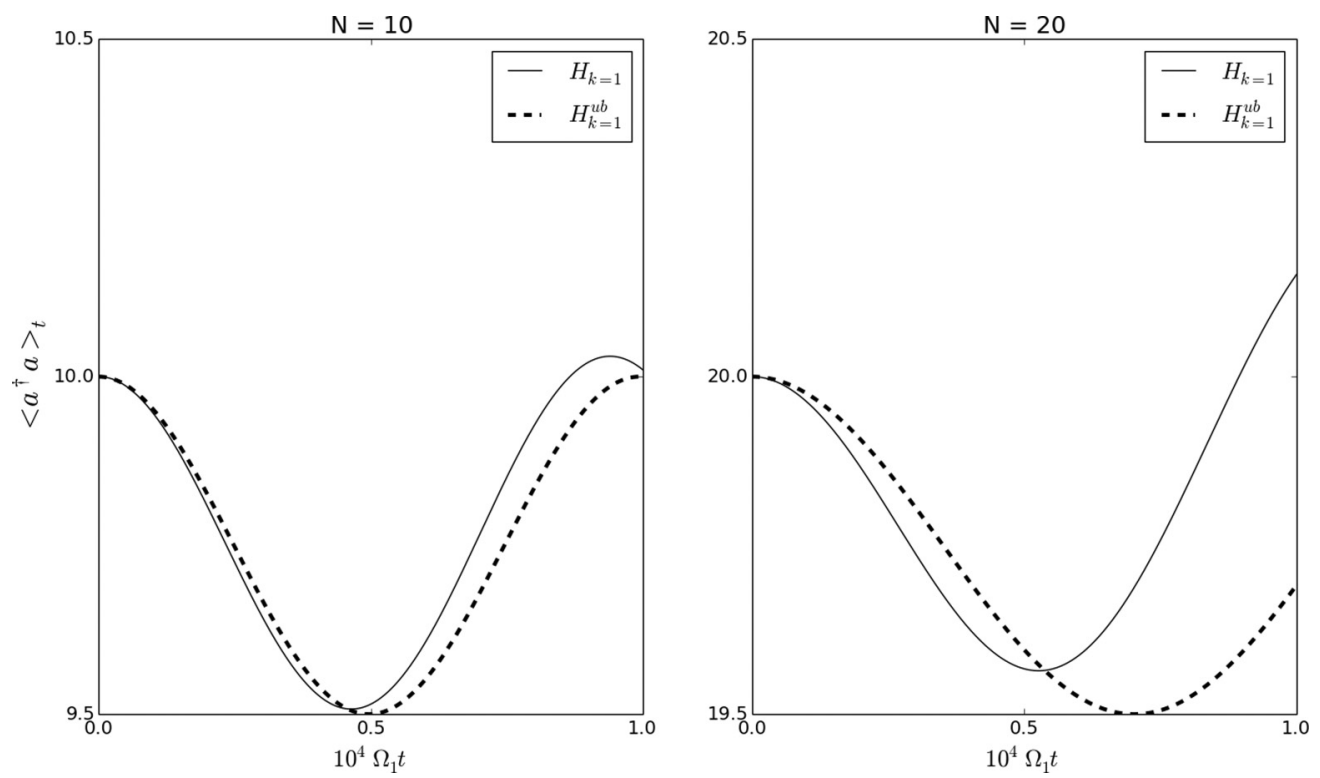

FIG. 1. The time evolution of the mean excitation $\left\langle a^{\dagger} a\right\rangle_{t}$ for the vibrational degrees of freedom computed from the full and the effective Hamiltonians in Eqs. (2) and (5) for $N=10$ and 20, starting from the electronic ground state and the vibrational Fock states $|10\rangle$ and $|20\rangle$, respectively, up to the time interval of $10^{4} \Omega_{1}^{-1}$. 
as discussed below. Expanding the operators $\mathbf{A}$ and $\mathbf{A}^{\dagger}$ in the Fock space basis we verify, under the above restrictions, that $\mathbf{A}^{\dagger}\left(\eta_{1}, \eta_{2}\right)|N\rangle \approx 0\left[\mathbf{A}\left(\eta_{1}, \eta_{2}\right)|N\rangle \approx 0\right]$, and consequently, for an initial vibrational state prepared within the upper-bound subspace ranging from $|0\rangle$ to $|N\rangle$, the Hamiltonian $H_{k= \pm 1}$ becomes

$$
H_{k= \pm 1}^{(u b)}=\sum_{n=0}^{N-1} \chi_{n}\left(|n\rangle\langle n+1| \sigma_{ \pm}+\text {H.c. }\right),
$$

where

$$
\chi_{n}=\sqrt{n+1}\left[1-\frac{\Omega_{2}}{\Omega_{1}}-\frac{1}{2 !}\left(\eta_{1}^{2}-\frac{\Omega_{2}}{\Omega_{1}} \eta_{2}^{2}\right) n\right] \Omega_{1} .
$$

To verify the validity of the engineered Hamiltonian (5), we plot in Fig. 1 the time evolution of the mean excitation $\left\langle a^{\dagger} a\right\rangle_{t}$ computed from the full and the effective Hamiltonians, in Eqs. (2) and (5), considering, as demanded above: $\Omega_{2} / \Omega_{1}=0.99, \eta_{2}=$ 0.01 , and $\eta_{1}^{2}=0.02 / N+\left(\Omega_{2} / \Omega_{1}\right) \eta_{2}^{2}$ for $N=10$ and 20 . We start from the electronic ground state and the vibrational Fock states $|10\rangle$ and $|20\rangle$ for the cases of $N=10$ and 20, respectively, to compute the expected excitations up to the time interval of $10^{4} \Omega_{1}^{-1}$ (exceedingly larger than the engineered relaxation time of the vibrational mode $\Gamma^{-1}$ ) where they start to slightly differ from each other. We note that in both cases, $N=10,20$, the upper bound cutoff mechanism works extremely well with the expectation value for the full Hamiltonian exceeding $N=10$ and 20 in about $2 \%$. Evidently, this error increases as time goes on.

We finally note that the same two-laser procedure leading to the upper-bound interaction (5) also leads to the lower-bound and the sliced interactions. 